\title{
An idea for detecting capture dominated Dark Stars
}

\author{
Fabio Iocco \\ Institut de Physique Théorique, CNRS, URA 2306 \&3 CEA/Saclay, F-91191 \\ Gif-sur-Yvette, France \\ Institut d'Astrophysique de Paris, UMR 7095-CNRS Paris, Universite' Pierre et Marie \\ Curie, boulevard Arago 98bis, 75014, Paris, France \\ email address: iocco@iap.fr
}

\begin{abstract}
I discuss an idea which could lead to a methodology for testing the effects of WIMP DM scattering and capture onto primordial stars. It relies on the effects of "life prolongation" of affected PopIII stars, that can slow down nuclear reactions by supporting their own structure with the energy produced by annihilating DM captured inside the star. This can lead to an alteration of the Pair Production SuperNova rate, which could constitute a peculiar signature of the existence of capture Dark Stars.
\end{abstract}

Key words: Early universe, dark matter, population III stars

\section{Prolegomena}

In the last two years there has been considerable activity to understand the effects of Weakly Interacting Massive Particles (WIMP) dark matter (DM) on the first stars to form in a $\Lambda$ CDM Universe, the Population III (PopIII). PopIII affected by WIMPs are commonly referred to as "Dark Stars"; in the following I will use this name for sake of simplicity, although preceded by gravitational and capture (dominated) to avoid any misunderstanding between completely different phenomena, that rely on diverse properties of the DM particle. In this Section, I will briefly summarize the state of the art of the field and identify the key-issues relevant to detection possibilities. Readers are addressed to the original references for a thorough discussion of the parameter dependence and details about the values adopted.

PopIII are thought to form by the very smooth collapse of the first $\mathcal{O}\left(10^{6} \mathrm{M}_{\odot}\right)$ DM and gas haloes to undergo non-linear collapse at redshifts 
$\mathrm{z} \lesssim 30$. Several groups, working at simulations with different techniques agree in describing the PopIII stellar formation as very different from local, lowredshift one, with remarkable effects such as the formation of a single, massive star per halo at its very center. Such objects are thought to live short lives $-\tau_{*} \sim 10^{6} \mathrm{yr}-$ and, depending on their mass, either directly collapse to a Black Hole or explode as Pair Production SuperNovae (PPSN), up to 100 times more powerful than normal typeII SN; for a review of the state of the art of the field, see e.g [1]. Weakly Interacting Massive Particles (WIMPs) are well motivated dark matter particle candidates, being the Lightest Supersymmetric Partner of the standard model, see e.g. [2] for reviews. Stable in models with $\mathrm{R}$-parity conservation, they bear the remarkable properties to be self-annihilating and weakly coupled to standard model particles.

In [3], the authors first showed that following the gas cooling and collapse, DM can concentrate in the center forming a steeper than NFW profile (to which I will refer in the following using the term "cusp"), and that this conjures in such a way that (for a wide range of DM masses and self-annihilation cross-sections) the heating induced by DM annihilation can overcome the feeble cooling of the pre-stellar cloud. The authors speculated this could generate a long-lasting equilibrium phase, and named the resulting astrophysical object a Dark Star; in the following I will refer to the condition in which gas cooling and DM heating rates equal as "transition point". This phase involves no scattering of DM off the gas: the WIMPs are gravitationally contracted by the gas collapse, and self-annihilate thus constituting an (additional) energy source; these effects are more efficient at late times during star formation, but yet far from the actual proto-star assembling. The results of [4], based on gas and DM profiles from three-dimensional simulations (without DM feedback on the chemistry or the equation of state of the system) confirmed the results of [3] up to the reach of the transition point, falling below simulation resolution afterwards.

In [5], the authors have studied the collapse phase of the gas preceding the transition point, investigating whether non-linear effects induced by the heating following DM annihilation could modify the properties of the gas before getting to it. They concluded that although DM annihilation does influence the properties of the gas even at intermediate states, changes are not so dramatic to modify the Jeans mass. Although this intermediate phase clearly deserves more study, and the behavior of the gas cooling and collapse in presence of DM annihilation need to be better understood, current results hint that the transition point can be reached without dramatic changes in 
presence of feedback between DM annihilation and the gas chemistry.

Once the formation of a proto-stellar object is achieved, the problem becomes to understand the evolution of an hydrostatic gas structure sustained by an underlying profile of self-annihilating DM, gravitationally coupled to the baryons; this has been addressed in [6, 7, 8] with different approaches. As of now, the results of the two groups seem to be discrepant: the authors of [7, 8] find that the DM concentrated inside the hydrostatic structure can sustain it against gravitational collapse for times of $\mathcal{O}\left(10^{6} \mathrm{yr}\right)$, thus letting the seed object accrete several hundreds of solar masses before igniting nuclear reactions; according to this scenario the main outcome of the gravitational Dark Star mechanism is a population of supermassive objects of $\mathcal{O}\left(800 \mathrm{M}_{\odot}\right)$. The authors of [6] do instead find a much less pronounced characteristic feature: a gravitational Dark Star is delayed in its normal path toward the Zero Age Main Sequence of up to no more than $10^{5} \mathrm{yr}$. This finding is obtained in absence of any gas accretion onto the seed object, it is however clear that even including accretion at the same rates used by [7, 8], the mass of the final object cannot be altered dramatically; this is also confirmed by recent findings of the authors of [6], so far released in several seminars and which will soon made public. Here I will rely on the latter scenario, in which the final outcome of the gravitational Dark Star mechanism, is a population not much dissembling the standard one, and slightly more massive, depending on parameters.

\section{Capture phase}

Before studying the effects of the gravitational phase on the baryonic structure, it was also realized by [9] that the peculiar formation of a PopIII star at the bottom of the gravitational potential -at the center of the formed DM cusp- would dramatically enhance the effects of WIMP capture (and subsequent annihilation) on the star, would it eventually form. It is to be noticed that this is a very different mechanism from the gravitational contraction, and it relies on weak interactions between baryons and WIMPs external to the star; a thorough review of the physics involved in this mechanism can be found in [10], whereas a more qualitative description of the differences between the two processes is summarized in [11]. Capture effects are irrelevant in early stages of evolution (and during the gravitational phase), typically starting to become relevant when the star is already on the Hayashi track, see [6]. The effects of WIMP capture from an external reservoir on the evolution of the first metal-free stars were studied in [6, 12, 13] and the results 
of the different groups are in remarkable agreement. The outcome can be summarized as follows: $i$ ) the lifetime of the star is prolonged (virtually up to higher than a Hubble time) in presence of high enough DM densities outside the star (environmental), as DM "fueling" slows down nuclear reactions and supports the star; ii) the time-prolonging effect is a very sharp function of the environmental DM density times the elastic scattering WIMP-baryon cross section, see e.g. Fig. 3 in [13], thus permitting to define a critical DM density (once picked the baryon-DM scattering cross-section value); iii) the final fate of the star $(\mathrm{SN} / \mathrm{BH})$ is unchanged, mainly because of the little effects of capture during the late stages of stellar evolution, see [12].

\section{An idea for detecting capture dominated Dark Stars}

The idea I propose relies on all of the properties mentioned above: a single PopIII star fueled by captured DM will live as long as the external DM density will stay above the critical DM density (see Fig. 6 in [6] for a dependence of the critical DM density as a function of stellar mass), or the star itself moves out of the DM reservoir. In [12] we estimated this time, $\Delta \tau_{\chi}$, as of $\mathcal{O}\left(10^{8} \mathrm{yr}\right)$, the typical timescale of a major halo merger at redshifts $z \sim 10$, relevant for PopIII stars. I will adopt this number -taken as a value averaged over the population - for practical purposes and comment more about it later. As a consequence of the life-prolongement, capture dominated Dark Stars will contribute differently than a standard PopIII to Reionization and stellar formation feedback in the early Universe; the consequences of this in terms of detectability of Dark Stars has been addressed with a first dedicated study by [14]. Another remarkable fact is that $\Delta \tau_{\chi}$ is much bigger than the typical lifetime of massive stars, $\tau_{*} \sim 10^{6} \mathrm{yr}$, and of the Hubble time at $\mathrm{z} \sim 10$. Therefore, whereas in a standard scenario the birth and death of massive PopIII are instantaneous on cosmological timescales, they will differ of $\Delta \tau_{\chi}$ in a capture Dark Star one. In terms of population, this may result in a delay of the PPSN rate, which will be more appreciable at higher redshift, where the ratio between $\Delta \tau_{\chi}$ and the Hubble time is bigger. In Figure 1, I show the effects of different $\Delta \tau_{\chi}$ on a PPSN rate. For this simple exercise I have taken the PopIII stellar formation rate (SFR) from the self-consistent reionization models from [15] and converted it into a PPSN rate by assuming a monochromatic initial mass function $(\mathrm{IMF}), \mathrm{M}_{*}=150 \mathrm{M}_{\odot}$; conversion of different SFRs and IMFs is trivial, and the shift effect does not change qualitatively. The most remarkable features are the absence (or drastic reduction) of PPSN

event until redshifts corresponding to Hubble times bigger than $\Delta \tau_{\chi}$, and 


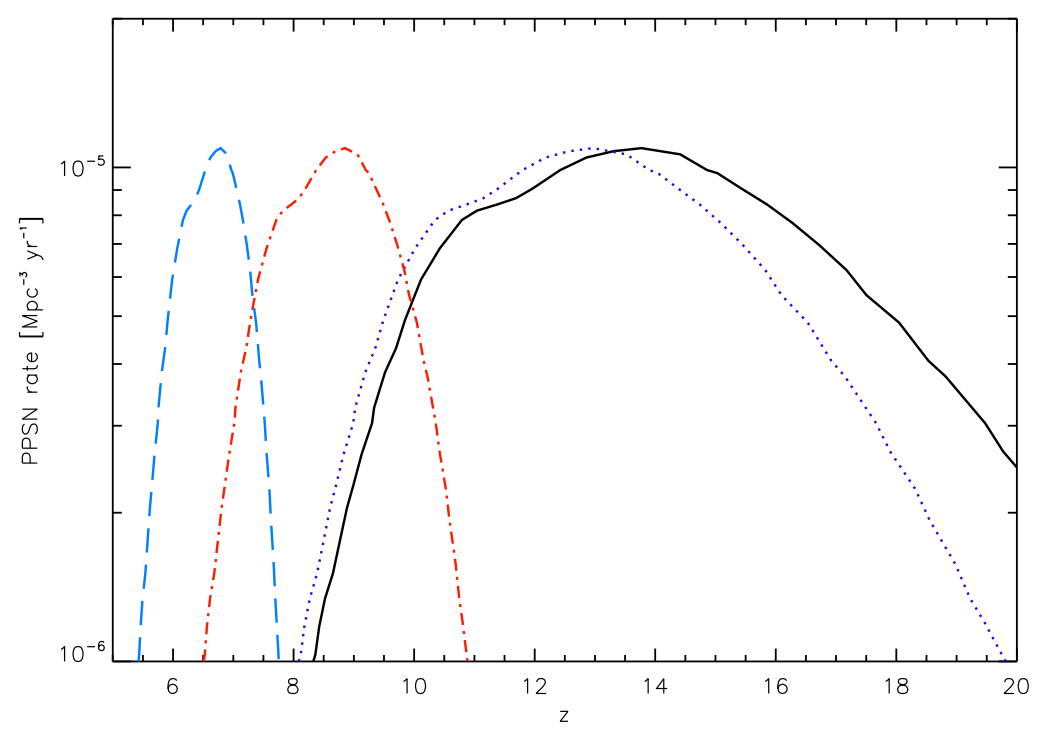

Figure 1: Shift of the PPSN rate as a consequence of a "life prolongement" effect due to DM capture on massive PopIII stars. The standard rate (solid line) is obtained by Fig. 3 in Choudhury \& Ferrara (2005), the dotted, dot-dashed, and dashed lines are for $\Delta \tau_{\chi}=5 \times 10^{7}, 5 \times 10^{8}, 10^{9} \mathrm{yr}$, respectively.

the appearance of events at lower redshifts, where there wouldn't be any in the standard case. However, the detectability of these features will be troubling: whereas the first relies on the capability to observe Pair Production SN at extremely high redhisfts, the latter, of intrinsic easier accessibility, would require (even more than the first) a detailed knowledge of the PPSN rate at low redshifts. Several authors have estimated it, see e.g. [16], but the results obtained are extremely sensitive to the properties of the Population III (as of now yet unaccessible with direct observations) and the quoted numbers vary of orders of magnitude. Hopefully we will have gathered more and enough knowledge to develop a methodology based on this idea by the JWST era, and then it will be time to discuss its feasibility and the disentanglement of the information; however, it is possible to briefly comment on some intrinsic constraints to its basis with elements at our hand as of now. In particular, $\Delta \tau_{\chi}$ will depend and be limited by: $i$ ) the self annihilation time of the DM cusp on which the star is "sitting"; ii) the depletion of an overcritical DM density by the star's own capture and use; iii) the deplacement of the star with respect to the cusp or the cusp disruption for friction, tidal effects 
etc; the latter can take place as a consequence of a merger, and its typical timescale at $\mathrm{z} \sim 10$ is of $\mathcal{O}\left(10^{8} \mathrm{yr}\right)$. Concerning the self-annihilation timescale of the DM cusp, by taking a $\rho_{D M} \sim 10^{11} \mathrm{GeV} / \mathrm{cm}^{3}$ (of the order of the critical densities for massive stars, by assuming a spin-dependent elastic scattering cross-section with baryons $\sigma_{0}=5 \times 10^{-39} \mathrm{~cm}^{2}$ ), and non exotic WIMP parameters such as mass $\mathrm{m}_{\chi}=100 \mathrm{GeV} / \mathrm{c}^{2}$ and $\langle\sigma v\rangle=3 \times 10^{-26} \mathrm{~cm}^{3} / \mathrm{s}$, one gets $\tau_{s a} \sim 10^{10} \mathrm{yr}$; this is the timescale on which the self-annihilation of the DM cusp outside the star will bring it below the critical density for life-prolonging effects on the star. The star also contributes to DM depletion by using it instead of nuclear burning to sustain itself: in one-dimensional models based on the Adiabatic Contraction, one typically finds that approximately $\mathcal{O}\left(1 \mathrm{M}_{\odot}\right)$ of DM is concentrated at supercritical densities around the star; for objects of $\mathrm{M}_{*} \sim 150 \mathrm{M}_{\odot}$ with characteristic luminosities of $\mathcal{O}\left(10^{6} \mathrm{~L}_{\odot} \sim 10^{39} \mathrm{erg} / \mathrm{s}\right)$ this reservoir takes approximately $\tau_{*}^{\chi} \sim 10^{8} \mathrm{yr}$ to be exhausted or lowered to undercritical densities (in principle accretion can bring more DM in the region surrounding the star, however the clue-issue is that the DM density has to stay supercritical to have the dramatic life-prolongement effect required for this framework). This back-of-the-envelope calculation based on central parameter values, show that $\Delta \tau_{\chi}$ has an upper limit of approximately $10^{10} \mathrm{yr}$, whereas a more reasonable value seems to lie in the range $10^{8} \mathrm{yr}$; our knowledge of this parameter will definitely benefit from dedicated high resolution simulations of the central regions of a primordial star forming halo, as well as of the behavior of a central baryonic object and of the DM during mergers of small halos at high redshifts.

I acknowledge fruitful conversations with A. Ferrara and M. Kamionkowski.

\section{References}

[1] V. Bromm, N. Yoshida, L. Hernquist and C. F. McKee, Nature 459, 49 (2009); "First Stars III", 2008, AIP Conf. Proc., 990, T. Abel, A. Heger, and B. W. 'O. Shea eds.

[2] G. Jungman, M. Kamionkowski and K. Griest, Phys. Rept. 267, 195 (1996); L. Bergstrom, Rep. Prog. Phys. 63, 793 (2000); G. Bertone, D. Hooper and J. Silk, Phys. Rept. 405, 279 (2005).

[3] D. Spolyar, K. Freese and P. Gondolo, Phys. Rev. Lett. 100, 051101 (2008). 
[4] A. Natarajan, J. C. Tan and B. W. O'Shea, Astrophys. J. 692, 574 (2009).

[5] E. Ripamonti, F. Iocco, A. Bressan, R. Schneider, A. Ferrara and P. Marigo, PoS(idm2008)075, arXiv:0903.0346 [astro-ph.CO].

[6] F. Iocco, A. Bressan, E. Ripamonti, R. Schneider, A. Ferrara and P. Marigo, Mon. Not. Roy. Astron. Soc. 390, 1655 (2008).

[7] K. Freese, P. Bodenheimer, D. Spolyar and P. Gondolo, Astrophys. J. 685, L101 (2008).

[8] D. Spolyar, P. Bodenheimer, K. Freese and P. Gondolo, arXiv:0903.3070 [astro-ph.CO].

[9] F. Iocco, Astrophys. J. 677, L1 (2008); K. Freese, D. Spolyar and A. Aguirre, JCAP 0811, 014 (2008).

[10] P. Scott, M. Fairbairn and J. Edsjo, Mon. Not. Roy. Astron. Soc. 394, $82(2009)$.

[11] F. Iocco, A. Bressan, E. Ripamonti, R. Schneider, A. Ferrara and P. Marigo, Procs. IAU Symposium 255 (2008) arXiv:0809.2417 [astro$\mathrm{ph}$.

[12] S. C. Yoon, F. Iocco and S. Akiyama, Astrophys. J. 688, L1 (2008).

[13] M. Taoso, G. Bertone, G. Meynet and S. Ekstrom, Phys. Rev. D 78, 123510 (2008).

[14] D. R. G. Schleicher, R. Banerjee and R. S. Klessen, Phys. Rev. D 79, 043510 (2009).

[15] T. R. Choudhury and A. Ferrara, Mon. Not. Roy. Astron. Soc. 361, 577 (2005); T. R. Choudhury and A. Ferrara, Mon. Not. Roy. Astron. Soc. 371, L55 (2006).

[16] S. M. Weinmann and S. J. Lilly, Astrophys. J. 624, 526 (2005); J. H. Wise and T. Abel, Astrophys. J. 629, 615 (2005); E. Scannapieco, P. Madau, S. Woosley, A. Heger and A. Ferrara, Astrophys. J. 633, 1031 (2005). 